AIMS Genetics, 6(4): 67-69.

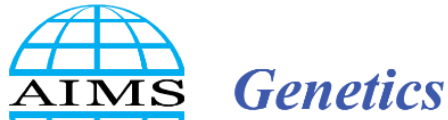

DOI: 10.3934 /genet.2019.4.67

Received: 27 September 2019

Accepted: 28 September 2019

Published: 30 September 2019

http://www.aimspress.com/journal/Genetics

\title{
Editorial
}

\section{A robust circular RNA-based prognostic signature for postoperative recurrence in stage II/III colon cancer}

Ji Ruan*

State Key Laboratory of Oncology in South China, Collaborative Innovation Center for Cancer Medicine, Sun Yat-sen University Cancer Center, Guangzhou 510060, China

* Correspondence: Ruanji@ sysucc.org.cn.

Abbreviations: circRNA: circular RNA; DFS: disease-free survival; HR: hazard ratio; CI: confidence interval; OS: overall survival

Colon cancer is one of the leading causes of mortality and morbidity worldwide [1], with a dramatic rise in incidence and mortality rates over the past 3 decades, both worldwide and in China [2]. Surgery is the principal therapy for early-stage colon cancer because it offers the only method for complete tumor removal, thereby chance for cure [3]. However, there is still proportions of colon cancer patients that develop recurrence even after radical resection [4]. Unfortunately, long term control of the recurrent colon cancer has been a difficult dilemma to tackle and the clinical outcomes of these patients are poor [5,6]. Adjuvant chemotherapy in colon cancer is able to complement curative surgery to reduce the risk of recurrence and death from relapsed or metastatic disease $[7,8]$. However, patient willingness to be adjuvantly treated has been limited due to its associated financial burden, treatment toxicity and debatable prolongation of survival that need to be carefully balanced against the associated-toxicity. Therefore, the identification of robust biomarkers to assess the risk of postoperative recurrence in colon cancer has been a longing need.

Circular RNAs (circRNAs), a recently discovered type of noncoding RNA, have a special circular structure formed by 3'- and 5'-ends linking covalently [9]. Because they do not have 5' or 3' ends, they are resistant to exonuclease-mediated degradation [10]. Therefore, circRNAs are characterized by their high stability, expression level, and evolutionary conservation. Recent literatures [11-13] have confirmed circRNAs as playing an important role in cancer initiation and progression. Additionally, they are differentially expressed in cancer tissues and circulation of cancer patients. Due to these characteristics, circRNAs have gained recognition as a promising novel biomarker for cancer [14]. However, till now little is known about circRNAs and their relationship with colon cancer. In a 
recent study published in EMBO Molecular Medicine, entitled "A circRNA signature predicts postoperative recurrence in stage II/III colon cancer", Ju et al. [15] identified and validated a circRNA-based signature that could improve postoperative prognostic stratification of patients with stage II/III colon cancer.

In that study, 437 colon cancer circRNAs were examined in tumoral and adjacent normal tissues. The authors found that 103 circRNAs were differentially expressed in recurrent colon cancer patients as compared to non-recurrent counterparts. Among them, 100 up-regulated circRNAs were validated in patients with stage II/III colon cancer to test whether they could be used as prognostic biomarkers. Four circRNAs, including hsa_circ_0122319, hsa_circ_0087391, hsa_circ_0079480, and hsa_circ_0008039, showed strong predictive values for disease-free survival (DFS) in the validation cohort. The following circRNA-based prognostic model was thereby generated:

\section{cirScore $=$}

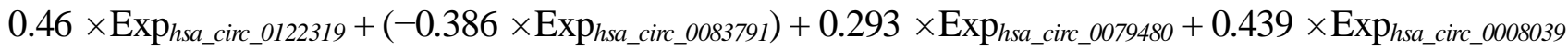

After adjusting for baseline clinicopathological factors, the cirScore was found suitable for serving as predictive biomarkers of postoperative recurrence in stage II/III colon cancer. Patients in the high-risk group (cirScore $\geq-0.323$ ) had poorer DFS (hazard ratio [HR], 2.89, 95\% confidence interval $[\mathrm{CI}], 1.37-6.09, P<0.001$ ) and overall survival (OS; HR, 4.22, 95\% CI, 1.61-11.03, $P<0.001$ ) than those in the low-risk group (cirScore $<-0.323$ ). Based on this circRNA-based prognostic model, the authors further established a nomogram which exhibited good performance for estimating the 3or 5-year DFS and OS.

To further explore the biological functions of hsa_circ_0122319, hsa_circ_0087391, and hsa_circ_0079480 (the abundance of hsa_circ_0008039 was found to be low in colon cancer cell line was therefore not further explored), the authors performed a series of functional in vitro and in vivo experiments. They found that suppression of these three circRNAs in colon cancer cells could significantly alter in vitro migration capacity. Additionally, the knockdown of hsa_circ_0079480 in colon cancer cells could both inhibit in vivo lung and liver metastasis.

The strengths of this study include the well-designed identification approaches, mature clinical data with clinical outcome and long-term follow-up, and well-characterized tissue samples for colon cancer. Limitations included that the prognostic model was only applicable for the patients with stage II/III colon cancer. In summary, this study identified a 4-circRNA-based expression signature as highly predictive for cancer recurrence in stage II/III colon cancer, with the potential of identification of high-risk early-stage colon cancer individuals who would benefit most from adjuvant chemotherapy.

\section{Conflict of interest}

The authors declare no conflict of interest.

\section{References}

1. Ferlay J, Colombet M, Soerjomataram I, et al. (2019) Estimating the global cancer incidence and mortality in 2018: GLOBOCAN sources and methods. Int J Cancer 144: 1941-1953.

2. Zhang B, Xie S, Yu I (2018) Differential incidence trends of colon and rectal cancers in Hong Kong: An age-period-cohort analysis. Cancer Commun 38: 42. 
3. Kim NK, Kim YW, Han YD, et al. (2016) Complete mesocolic excision and central vascular ligation for colon cancer: Principle, anatomy, surgical technique, and outcomes. Surg Oncol 25: 252-262.

4. Schmoll HJ, Van Cutsem E, Stein A, et al. (2012) ESMO Consensus Guidelines for management of patients with colon and rectal cancer. A personalized approach to clinical decision making. Ann Oncol 23: 2479-2516.

5. Chen T, Huang Y, Wang G (2017) Outcome of colon cancer initially presenting as colon perforation and obstruction. World J Surg Oncol 15: 164.

6. Peng J, Zhang R, Zhao Y, et al. (2017) Prognostic value of preoperative prognostic nutritional index and its associations with systemic inflammatory response markers in patients with stage III colon cancer. Chin J Cancer. 36: 96.

7. Dienstmann R, Salazar R, Tabernero J (2015) Personalizing colon cancer adjuvant therapy: Selecting optimal treatments for individual patients. J Clin Oncol 33: 1787-1796.

8. Yan L, Zhang W (2018) Precision medicine becomes reality-tumor type-agnostic therapy. Cancer Commun 38: 6.

9. Wilusz JE, Sharp PA (2013) Molecular biology. A circuitous route to noncoding RNA. Science 340: 440-441.

10. Jeck WR, Sorrentino JA, Wang K, et al. (2013) Circular RNAs are abundant, conserved, and associated with ALU repeats. Cold Spring Harbor Lab Press 19: 141-157.

11. Fang L, Du W, Lyu J, et al. (2018) Enhanced breast cancer progression by mutant p53 is inhibited by the circular RNA circ-Ccnb1. Cell Death Differ 25: 2195-2208.

12. Yang Q, Du WW, Wu N, et al. (2017) A circular RNA promotes tumorigenesis by inducing c-myc nuclear translocation. Cell Death Differ 24: 1609-1620.

13. Zhang M, Zhao K, Xu X, et al. (2018) A peptide encoded by circular form of LINC-PINT suppresses oncogenic transcriptional elongation in glioblastoma. Nature Commun 9: 4475.

14. Meng S, Zhou H, Feng Z, et al. (2017) CircRNA: Functions and properties of a novel potential biomarker for cancer. Mol Cancer 16: 94.

15. Ju H, Zhao Q, Wang F, et al. (2019) A circRNA signature predicts postoperative recurrence in stage II/III colon cancer. EMBO Mol Med 2019: e10168.

(C) 2019 the Author(s), licensee AIMS Press. This is an open access article distributed under the terms of the Creative Commons Attribution License (http://creativecommons.org/licenses/by/4.0) 